\title{
BMJ Open Acute estradiol and progesterone therapy in hospitalised adults to reduce COVID-19 severity: a randomised control trial
}

\author{
Dragana Lovre (D) , ${ }^{1,2,3}$ Kristin Bateman, ${ }^{4}$ Mya Sherman, ${ }^{5}$ Vivian A Fonseca, ${ }^{1,2}$ \\ John Lefante, ${ }^{6}$ Franck Mauvais-Jarvis ${ }^{1,2,3}$
}

To cite: Lovre D, Bateman K, Sherman $\mathrm{M}$, et al. Acute estradiol and progesterone therapy in hospitalised adults to reduce COVID-19 severity: a randomised control trial. BMJ Open 2021;11:e053684. doi:10.1136/ bmjopen-2021-053684

- Prepublication history for this paper is available online. To view these files, please visit the journal online (http://dx.doi. org/10.1136/bmjopen-2021053684).

Received 20 May 2021 Accepted 01 November 2021

Check for updates

(C) Author(s) (or their employer(s)) 2021. Re-use permitted under CC BY-NC. No commercial re-use. See rights and permissions. Published by BMJ.

For numbered affiliations see end of article.

Correspondence to Dr Franck Mauvais-Jarvis; fmauvais@tulane.edu

\section{ABSTRACT}

Introduction As of November 2021, COVID-19 has killed more than 5 million people globally, including over 750000 in the USA. Apart from corticosteroids, most available therapeutic options are at best marginally efficient in reducing disease severity and are extremely expensive. The systematic investigation of clinically approved drugs is a priority to determine what does mitigate disease severity. Oestradiol (E2) and progesterone (P4) produce a state of anti-inflammatory immune responses and immune tolerance, and enhanced antibody production. The goal of this trial is to evaluate the efficacy of a short E2 and P4 therapy, in addition to standard of care (SOC), in mitigating disease severity in COVID-19 hospitalised patients. Methods and analysis Phase 2, randomised, double blind, placebo-controlled, single-centre trial. Patients hospitalised for confirmed COVID-19, with scores 3-5 on the 9-point WHO ordinal scale are randomised between two arms: (1) Oestradiol cypionate intramuscular (IM) and micronised progesterone oral (PO), in addition to SOC, and (2) placebo, in addition to SOC. The primary outcome is the proportion of patients improving to scores 1 or 2 on the WHO scale through day 28 . Secondary outcomes include length of hospital stay, duration of mechanical ventilation, cause of death, readmission rates, change in inflammatory biomarkers between admission and occurrence of primary endpoint, and adverse events. Study sample size will be up to 120 participants. The trial is currently recruiting subjects.

Ethics and dissemination The sponsor of this study is the Center of Excellence in Sex-Based Biology \& Medicine at Tulane University, New Orleans, Louisiana, USA. Ethical approval was obtained from the Tulane institutional review board on 14 May 2021. The study was reviewed by the US Food and Drug Administration and granted Investigational New Drug \#152 499. Results of the study will be submitted for publication in a peer-reviewed journal.

Trial registration number NCT04865029; Pre-results.

\section{INTRODUCTION}

As of November 2021, COVID-19 has already killed over 5 million people worldwide. ${ }^{1}$ Although the vaccination campaign is ramping up in the USA, vaccination hesitancy represents up to $25 \%-30 \%$ of the population, ${ }^{2}$
Strengths and limitations of this study

The trial will include men and women.

- Oestradiol cypionate (E2) and progesterone (P4) decrease production of proinflammatory cytokines and enhance B-cell-mediated antibody production.

- The advantage of repurposing E2 and P4 comes from decades of accumulated knowledge regarding their clinical efficacy and toxicity.

- E2 and P4 are inexpensive and available in hospitals worldwide.

- A randomised, placebo-controlled trial is an efficient way to determine if a short systemic E2 and P4 therapy, administered early to hospitalised COVID-19 patients in addition to standard of care (SOC) can reduce the severity of outcomes compared with SOC alone.

and hospitalisations and deaths are still high. Apart from corticosteroids, ${ }^{3}$ most available therapeutic options are at best marginally efficient in reducing disease severity and mortality and are extremely expensive. ${ }^{4-6}$ Therefore, the systematic investigation of clinically approved drugs is still a priority in order to determine what compounds could be repurposed to mitigate disease severity, and invest resources to go to full-scale production. Our current understanding of the disease is that COVID-19 deaths result from an inappropriate immune response with outpouring of proinflammatory chemokines leading to lung infiltration and hyperactivation of monocytes and macrophages producing proinflammatory cytokines (cytokine storm), resulting in lung oedema, reduced gas exchange, and ultimately, leading to acute respiratory distress syndrome and multiorgan failure. ${ }^{7-14}$ Men with COVID-19 have a uniformly more severe outcome than women. In series from China, Europe and the USA, COVID-19 mortality was consistently 1.5 -fold 
to 2-fold higher in men than in women, suggesting that female biological sex is protecting women from COVID-19 mortality. ${ }^{15-20}$ It is established that women exhibit heightened immune responses to viral infections compared with men, ${ }^{21}$ which is at least partially due to the genetic benefit of gene dosage in X-linked immuneresponse genes. Ovarian steroids, however, also play a protective role. The analysis of electronic health records of over 68000 COVID-19 patients revealed that oestrogen therapy is associated with more than $50 \%$ reduction in mortality. ${ }^{22}$ The main female steroids, $17 \beta$-oestradiol (E2) and progesterone (P4) exhibit potent immunomodulatory and anti-inflammatory actions via oestrogen and progesterone receptors expressed in all immune cells, including epithelial cells, macrophages, dendritic cells, CD4 +and CD8+lymphocytes and B cells. ${ }^{21} 23$ P4 also acts partially via the glucocorticoid receptor. Together $\mathrm{E} 2$ and $\mathrm{P} 4$ produce a state of decreased innate immune cells production of proinflammatory cytokines, enhanced $\mathrm{T}$ cells anti-inflammatory responses and immune tolerance, and enhanced B-cell-mediated antibody production. $^{212425}$ The scientific premise of this trial is that in hospitalised COVID-19 patients, a short and early E2 and P4 therapy, in addition to standard of care (SOC; the National Institutes of Health (NIH) COVID-19 Treatment Guidelines Panel recommends the use of dexamethasone for up to 10 days or until hospital Discharge as SOC for the treatment of hospitalised COVID-19 patients), will prevent or mitigate the cytokine storm, and prevent severe outcomes, and without side effects. ${ }^{25}$ Therefore, it will provide steroid immunomodulation without immunosuppression. The advantage of repurposing E2 and P4 is the depth of knowledge regarding their clinical efficacy and toxicity that has accumulated from decades of clinical and basic studies. E2 and P4 are widely available in hospitals, inexpensive, manufacturable to scale and can be prescribed immediately.

\section{METHODS AND ANALYSIS}

Study design, randomisation, intervention and study calendar

Phase 2, randomised, double blind, placebo-controlled, single centre trial designed to determine to what extent a short systemic E2 and P4 therapy, administered early to hospitalised COVID-19 positive patients of both sexes, in addition to SOC, can reduce the severity of symptoms and outcomes compared with SOC alone.

Hospitalised patients with COVID-19 with scores 3-5 on the 9-point WHO ordinal scale ${ }^{26}$ are randomised to a 5 day treatment between two arms:

1. Oestradiol cypionate (depo-oestradiol) $5 \mathrm{mg}$ via intramuscular injection on day 1 and micronised progesterone $200 \mathrm{mg}$ administered orally, daily for 5 days, in addition to SOC.

2. Intramuscular and oral placebos in addition to SOC.

We chose E2 administration via IM injection to avoid first-pass liver metabolism and change in coagulation markers while we chose $\mathrm{P} 4$ oral $(\mathrm{PO})$ preparation due to ease of administration and low side effect profile. Drug will be supplied by Tulane Medical Center Research Pharmacy. The manufacturers of the study drugs are: Pfizer for E2 (NDC: 0009-0271-01) and VIRTUS for P4 (NDC: 69543-375-10).

An independent biostatistician will provide a random number table to the unblinded pharmacist for randomisation. The random number table is generated based on the total number of subjects to be enrolled in a 1:1 ratio for placebo vs $\mathrm{E} 2 / \mathrm{P} 4$. The unblinded pharmacist will use the random number table in sequential order for randomisation assignment as subjects are enrolled. The research team will remain blinded to the randomisation assignment throughout the study. Unblinding will only be permissible in case of adverse events (AEs).

Study design and visit schedule are shown in figure 1.

\section{Study implementation, recruitment, population and eligibility criteria}

Participants are recruited at admission by the medical staff of the Department of General Internal Medicine and Geriatrics at Tulane Medical Center (Tertiary care academic hospital), under the leadership of KB (coinvestigator (Co-Is)) who identifies participants meeting study eligibility criteria from their admission list. KB assesses eligibility at screening visit, and notifies the research coordinators of eligible participants. Eligible subjects are enrolled by the research coordinators to partake in a randomised scheme to receive the 5-day active treatment or placebo. The Research Pharmacist at Tulane Medical Center assigns participants to the intervention. We will recruit up to 120 participants hospitalised at Tulane Medical Center with mild to severe COVID-19 (WHO ordinal scale score 3-5, table 1) confirmed by SARSCoV-2 PCR test.

\section{Inclusion criteria}

1. Hospitalisation for COVID-19 (WHO Ordinal scale score 3-5, table 1) confirmed by SARS-CoV-2 PCR at Tulane Medical Center in the Department of General Internal Medicine and Geriatrics.

2. Respiratory symptoms (fever, shortness of breath or cough) or abnormal lung exam or chest imaging characteristic of mild to severe COVID-19 pneumonia.

3. Patient and/or legally authorised representative (LAR) agrees to comply with study procedures and the collection of blood samples per protocol.

4. Patient and/or LAR agrees to be placed on prophylactic dose of anticoagulation for prevention of deep venous thrombosis (DVT) (if necessary).

5. Patient or legally authorised representative has signed informed consent.

6. Women of childbearing age with a negative pregnancy test on admission.

\section{Exclusion criteria}

1. Patient under 18 years of age. 


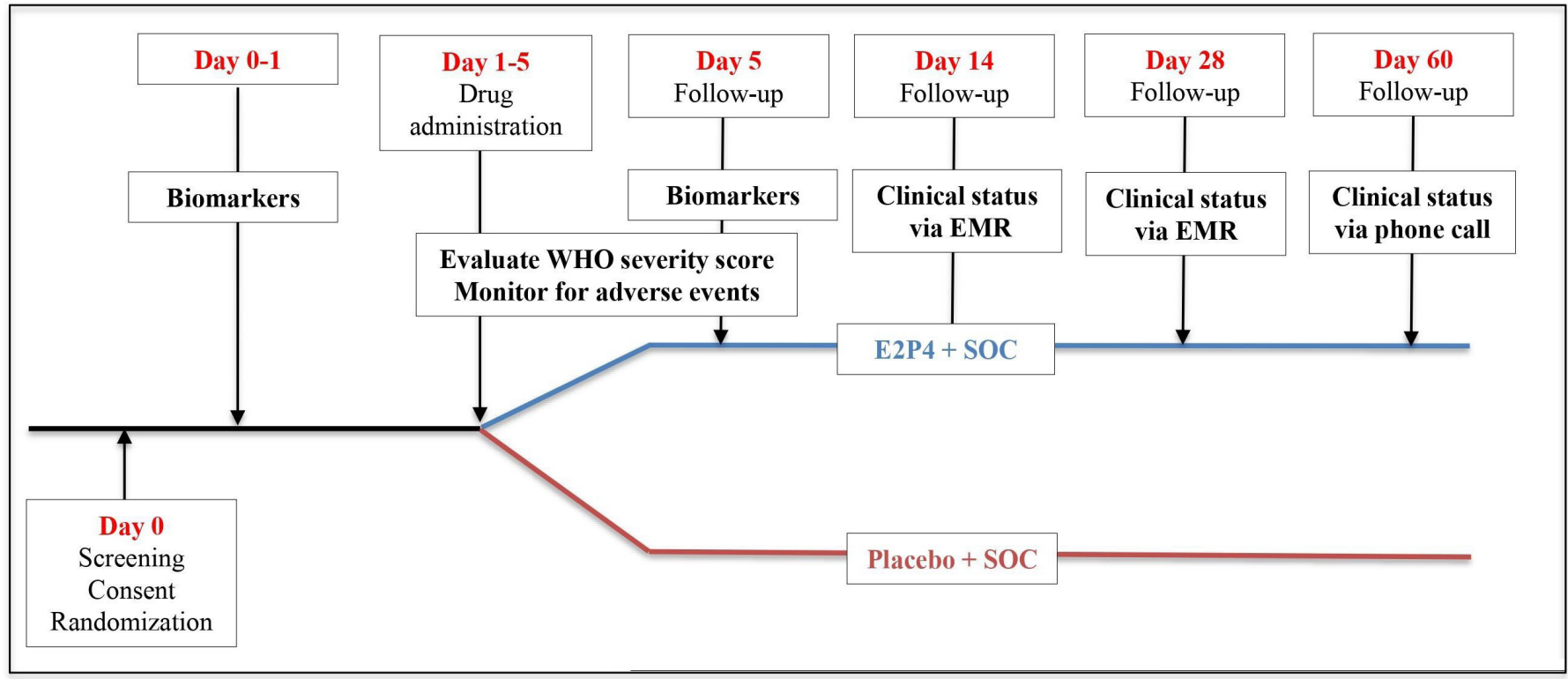

Figure 1 Study design. E2 $5 \mathrm{mg}$ IM injection will be administered once on day 1 and P4 $200 \mathrm{mg}$ will be administered daily orally $(\mathrm{PO})$ day $1-5$. Clinical status follow-up via electronic medical record (EMR) review will be performed day 14 th and day 28th. Clinical status for the end of study visit will be on day 60 via telephone and EMR review. E2, oestradiol cypionate; IM; Intramuscular; P4, progesterone (oral); SOC, standard of care.

2. Critically ill COVID-19 (respiratory failure requiring intubation and mechanical ventilation, shock, multiorgan failure).

3. Pregnant women confirmed by pregnancy test.

4. Women within 6 weeks of post partum.

5. Patient included in another COVID-19 trial (excluding hydroxychloroquine and dexamethasone).

6 . Women already treated by oestrogen and or progestogen therapy 2 weeks prior to admission.

7. Men already treated by testosterone therapy prior to admission.

8. History of breast or endometrial cancer.

\begin{tabular}{|c|c|c|}
\hline Patient state & Descriptor & Score \\
\hline Uninfected & $\begin{array}{l}\text { No clinical or virological evidence of } \\
\text { infection }\end{array}$ & 0 \\
\hline \multirow[t]{2}{*}{ Ambulatory } & No limitation of activities & 1 \\
\hline & Limitation of activities & 2 \\
\hline \multirow{2}{*}{$\begin{array}{l}\text { Hospitalised } \\
\text { mild disease }\end{array}$} & Hospitalised, no oxygen therapy & 3 \\
\hline & Oxygen by mask or nasal prongs & 4 \\
\hline \multirow{3}{*}{$\begin{array}{l}\text { Hospitalised } \\
\text { severe } \\
\text { disease }\end{array}$} & $\begin{array}{l}\text { Non-invasive ventilation or high-flow } \\
\text { oxygen }\end{array}$ & 5 \\
\hline & Intubation and mechanical ventilation & 6 \\
\hline & $\begin{array}{l}\text { Ventilation +additional organ } \\
\text { support-pressors, renal replacement } \\
\text { therapy, extracorporeal membrane } \\
\text { oxygenation }\end{array}$ & 7 \\
\hline Dead & Death & 8 \\
\hline
\end{tabular}

*Table 1 was adapted from reference 26.
9. Abnormal genital bleeding.

10. Active or recent (eg, within the past year) stroke or myocardial infarction.

11. History of blood clots including deep vein thrombosis related to clotting disease, or pulmonary emboli (prior to hospitalisation).

12. History of liver dysfunction or disease.

13. Patients with end-stage renal disease.

14. Patients taking inhibitors of CYP3A4 such as erythromycin, clarithromycin, ketoconazole, itraconazole and ritonavir.

15. Patients taking St. John's Wort preparations (hypericum perforatum), phenobarbital, carbamazepine and rifampin.

16. Patients within 6 weeks of major orthopaedic surgery.

\section{Safety measures and monitoring}

We will monitor each subject for AEs/serious AEs (AEs/ SAEs) daily while the subjects are receiving daily therapy (figure 2). If they develop a grade 3 or $4 \mathrm{AE}$, we will discontinue the study drug. No dose adjustments will be made. We will also follow up in person or by telephone for all subjects for any adverse AEs/SAEs and mortality at day 60 .

AEs will be graded according to Common Terminology Criteria for Adverse Events Version5 and taken care with the corresponding specialised clinical management of the SAE.

Grade 1 AEs (asymptomatic or mild symptoms; clinical or diagnostic observations only; intervention not indicated) or grade 2 AEs (moderate; minimal, local or noninvasive intervention indicated; limiting age appropriate instrumental activity of daily living): such as breast tenderness, nausea, vomiting, bloating, stomach cramps, 


\begin{tabular}{|l|c|c|c|c|c|c|c|c|c|}
\hline \multirow{2}{*}{ Surveillance } & \multicolumn{7}{|c|}{ Days following initiation of treatment } \\
\hline Pregnancy test* & 0 & 1 & 2 & 3 & 4 & 5 & 14 & 28 & 60 \\
\hline Injection site & $\mathrm{X}$ & & & & & & & & \\
\hline E2 side effects & & $\mathrm{X}$ & $\mathrm{X}$ & $\mathrm{X}$ & $\mathrm{X}$ & $\mathrm{X}$ & & & \\
\hline P4 side effects & & $\mathrm{X}$ & $\mathrm{X}$ & $\mathrm{X}$ & $\mathrm{X}$ & $\mathrm{X}$ & $\mathrm{X}$ & & \\
\hline DVT symptoms & & $\mathrm{X}$ & $\mathrm{X}$ & $\mathrm{X}$ & $\mathrm{X}$ & $\mathrm{X}$ & $\mathrm{X}$ & & \\
\hline MI symptoms & & $\mathrm{X}$ & $\mathrm{X}$ & $\mathrm{X}$ & $\mathrm{X}$ & $\mathrm{X}$ & $\mathrm{X}$ & $\mathrm{X}$ & $\mathrm{X}$ \\
\hline Stroke symptoms & & $\mathrm{X}$ & $\mathrm{X}$ & $\mathrm{X}$ & $\mathrm{X}$ & $\mathrm{X}$ & $\mathrm{X}$ & $\mathrm{X}$ & $\mathrm{X}$ \\
\hline Fluid retention** & & $\mathrm{X}$ & $\mathrm{X}$ & $\mathrm{X}$ & $\mathrm{X}$ & $\mathrm{X}$ & $\mathrm{X}$ & & $\mathrm{X}$ \\
\hline
\end{tabular}

Figure 2 Schedule of safety assessments. Study subjects will be monitored for symptoms daily while receiving the study drugs day 1-5. Relevant safety assessments otherwise will be performed via chart review on day 14th and 28th. End of study visit safety assessment will be on day 60 via telephone and EMR review. ${ }^{*}$ Women in reproductive age. ${ }^{* *}$ Patients with renal impairment. DVT, deep venous thrombosis; MI, myocardial infarction; EMR; electronic medical record; E2, oestradiol cypionate; P4, progesterone.

headaches, vaginal itching, abnormal uterine bleeding will not require discontinuation of treatment.

Grade 3 SAEs like DVT and 4 (life-threatening) SAEs such as MI, stroke, anaphylaxis will be managed by treatment discontinuation (except SOC), and appropriate specialised clinical management of the SAE.

The trial is a 5-day intervention during hospitalisation and therefore we do not expect problems with adherence. The study team members who are administering the trial drugs will call the Principal Investigator (PI) or the Co-Principal Investigators (Co-Is) in circumstances when subjects have questions or concerns to be addressed in a timely manner to improve adherence.

Study investigators have formed an independent data safety and monitoring board (DSMB) to monitor study safety. The DSMB consists of four board members who are clinicians and researchers with the experience necessary to interpret the data and ensure patient safety. Specifically, the board members are knowledgeable about COVID-19 and the treatment involved in this study. The DSMB includes, an infectious disease specialist, an endocrinologist, a haematologist-oncologist, a women's health specialist and a biostatistician. The initial DSMB meeting occurred before the start of the trial to discuss the protocol and guidelines for monitoring the study. At this meeting, guidelines were defined for stopping the study for safety concerns (Study stopping rules) and where relevant, for efficacy based on plans specified in the protocol. The DSMB will meet on a quarterly basis. After each meeting, the DSMB will provide the PI with a letter describing the results of their review and their recommendations for the continuation of the study.

\section{Outcome measures}

For all randomised patients according to inclusion criteria section above, demographic and clinical variables, including sex, age, race/ethnicity, body mass index (BMI), symptoms, vital signs, blood pressure, respiratory rate, temperature, oxygen saturation, chronic comorbidities through diagnosis codes, medications, clinical course during hospitalisation (described in primary outcome below) will be reviewed in the patients' medical charts. Laboratory measurements will include, when available, complete blood count (CBC), blood chemistry, liver function test, creatinine, blood urea nitrogen test, lactate, troponin, ferritin, $\mathrm{C}$ reactive protein (CRP), procalcitinin, brain-type natriuretic peptide, D-dimer and interleukin-6.We will review the participants' medical records for up to 60 days after hospital discharge.

\section{Primary outcome}

For all randomised patients with baseline inclusion criteria, WHO 9-point ordinal scale scores 3-5, the primary efficacy end point will be the proportion of patients who improve to scores 1 or 2 on the WHO ordinal scale (table 1) through day 28.

\section{Secondary outcomes}

For all randomised patients, the following secondary outcomes will be assessed at day 14, 28 and 60 . 
Assessment at day 14 and 28 will be performed by electronic medical records review, while assessment at day 60 will be performed via telephone call directly to the study subjects.

1. Length of hospital stay.

2. Duration of mechanical ventilation.

3. Date and cause of death.

4. Readmission.

5. Change in biological markers below between admission and occurrence of primary endpoint (two values at least 2 days apart).

6. Grade 3 and 4 AEs.

7. SAEs.

\section{Biological markers}

Obtained from SOC:

Inflammation: neutrophil:lymphocyte ratio, CRP, ferritin, procalcitonin.

Hypercoagulability: D-dimers, fibrinogen.

Tissue injury: troponin, alanine transaminase, aspartate transaminase, lactate dehydrogenase.

Metabolome, proteome, peripheral blood mononuclear cells (PBMCs).

\section{Data collection and management}

The collection of routine blood samples and patient monitoring will be conducted as part of the SOC procedures that are used at Tulane Medical Center for COVID-19 patients. The collection of blood samples (CBC with differential, blood chemistry, inflammation markers) is to monitor disease severity and is independent from this protocol, but we will use the data in our analysis. Women of childbearing age will have a pregnancy test on admission.

An additional blood sample will be collected at admission and after 5 days of treatment (about $5-25 \mathrm{~mL}$ of blood at these two time points). This blood sample will be stored in FM-J lab for (1) isolation of PBMC to assess the effect of treatment on immune cells populations by flow cytometry, and PBMC gene expression by RNA-Seq and (2) metabolomics profiling to determine metabolic and molecular signatures of the efficacy of treatment. Deidentified serum and PBMC frozen samples will be transferred to the University of Michigan Metabolomics Core to perform untargeted metabolomic analyses. The serum and PBMC from collected blood samples will be used to perform multidimensional analysis in the Center for Translational Research in Infection and Inflammation at Tulane University School of Medicine. Blood samples will be stored for up to 3 years.

The majority of clinical data, including all blood sample collection and assessment of severity of illness, will occur while the patient is hospitalised, which should minimise patient non-adherence. The only information to be collected after discharge will be a telephone call to assess clinical status at day 60. All available data will be collected and assessed into our analysis. If patients choose to discontinue from the study after day 1 (when they will have already received oestradiol if in the treatment group), we will separate the analysis from the treatment group as they will not have completed the progesterone therapy.

All data and research files will be de-identified and stored using the secure web application Research Electronic Data Capture (REDCap). REDCap is protected by a login as well as encryption. All data for this study will be electronic; there will be no paper records. Enrolled subjects will be assigned a sequential study ID (ie, 001, 002 , etc), that is, linked to their medical record number. A link between the study ID numbers and medical record number will be kept in a separate file from the deidentified database and stored securely in REDCap. To promote data quality, two different individuals will perform double data entry. Subsequently, comparison of the two records will be done and any non-matching data will be correctly identified and entered. Only the institutional review board (IRB)-approved study investigators will have access to the data and link. The PI will store all study research records and data post study closure, as required by NIH and University data retention policies.

\section{Statistical considerations \\ Sample size}

Sample size calculations are based on $80 \%$ Power, using a two-sided Pearson's $\chi^{2}$ test for proportion differences at the $5 \%$ significance level. It is estimated that between $60 \%$ and $70 \%$ of hospitalised patients with mild or severe disease will improve clinically and become ambulatory after standard treatment (WHO ordinal scale score $1-2$, table 1)). Table 2 presents the sample sizes that are required in each treatment group to detect a significant difference in the per cent of hospitalised patient leading to an ambulatory disease (shift from category 3-5 to category $1-2$ in $20 \%, 25 \%, 30 \%$ or $35 \%$ of hospitalised patients after treatment with E2 and P4). Efforts will be made to recruit 60 patients in each treatment group in order to stratify by disease severity, age and gender.

\begin{tabular}{lll}
\hline Table 2 & Sample size calculations & \\
\hline Treatment & $\begin{array}{l}\text { Per cent leading to a } \\
\text { scale 1-2 }\end{array}$ & $\begin{array}{l}\text { Sample } \\
\text { size }\end{array}$ \\
\hline SOC & 60 & 32 \\
SOC+E2+P4 & 90 & 32 \\
SOC & 60 & 22 \\
SOC+E2+P4 & 95 & 22 \\
SOC & 70 & 62 \\
SOC+E2+P4 & 90 & 62 \\
SOC & 70 & 36 \\
SOC+E2+P4 & 95 & 36
\end{tabular}

E2, oestradiol cypionate; P4, micronised progesterone; SOC, standard of care. 
Table 3 Interim analysis sample size calculation for a binary endpoint

\begin{tabular}{lll}
\hline Stage & $\mathbf{1}$ & $\mathbf{2}$ \\
\hline Information rate & $50 \%$ & $100 \%$ \\
\hline Efficacy boundary (z-value scale) & 2.963 & 1.969 \\
\hline Overall power & 0.1641 & 0.8000 \\
No of subjects & 61.8 & 123.7 \\
Cumulative alpha spent & 0.0031 & 0.0500 \\
Two-sided local significance level & 0.0031 & 0.0490 \\
Lower efficacy boundary (t) & -0.377 & -0.172 \\
\hline Upper efficacy boundary (t) & 0.277 & 0.148 \\
\hline
\end{tabular}

Sequential analysis with a maximum of 2 looks (group sequential design). The sample size was calculated for a two-sample test for rates (two sided), $\mathrm{HO}$ : $\mathrm{pi}(1)-\mathrm{pi}(2)=0, \mathrm{H} 1$; treatment rate $\mathrm{pi}(1)=$ 0.9 , control rate $\mathrm{pi}(2)=0.7$. $(\mathrm{t})$ : approximate treatment effect scale.

\section{Interim analysis}

One and only interim analysis will be performed at $50 \%$ study completion (after 60th subject end of study visit) to determine to what extent a strong treatment efficacy warrants stopping the trial early. Table 3 presents interim sample sizes and required $\mathrm{Z}$ test statistics for comparing two proportions at each of stage of an O'Brien-Fleming alpha-spending sequential design. ${ }^{27}$ Based on $80 \%$ power and an overall $5 \%$ significance level, if the interim analysis for the first 60 enrolled patients results in differences that are more than $2.96 \mathrm{SD}$ from 0 , the trial will end, and treatment efficacy will be concluded. Otherwise, the trial will enrol the total 120 patients.

\section{Statistical analysis}

For all subjects who have completed the study, clinical outcomes and demographic characteristics will be summarised and presented separately for the treatment group and the control group, as well as overall for all patients. Categorical variables, such as the primary outcome, readmission, as well as race and sex will be summarised with counts and percentages. Quantitative outcomes, such as length of stay, change in biological markers, age and BMI will be summarised with means, medians, (standard deviation) SD and interquartile ranges (IQRs). Univariate analyses will be performed as the primary method to assess the unadjusted effect of treatment on the primary outcome and on readmission rate using the Pearson $\chi^{2}$ test. Differences in average length of stay and in change in biological markers will be assessed with either a two-sample t test or the MannWhitney non-parametric test. Multivariate analyses will be performed to compare treatment to control responses while adjusting for various co-factors. Multiple logistic regression will be used as supportive method to test for significant differences in the proportion with the primary outcome, between the active treatment and placebo groups, while adjusting for age, race, sex, BMI and other potential cofactors. For the secondary outcomes, multiple logistic regression will also be used to test for significant differences in the proportion readmits, between the active treatment and placebo groups, and multiple linear regression will be used to test for significant differences in average length of stay and changes in biological markers, between the treatment and SOC groups, while adjusting for potential influencing co factors.

\section{Patient and public involvement}

No patients were involved in the design or implementation of this study.

\section{ETHICS AND DISSEMINATION \\ Ethics and safety approval}

Ethical approval was obtained from the IRB at Tulane University Clinical Translational Unit on 14 May 2021. Future amendments to the protocol by the study investigators will be submitted to the IRB. Implementation of changes in the study will only be made after the IRB approval is received. Accordingly, trial participants, trial registries and trial staff will be informed in a timely manner.

The study has been reviewed by the US Food and Drug Administration and granted Investigational New Drug \#152499.

\section{Informed consent}

The consent process will be administered to the patient (or LAR in the case that the patient is unable to consent) prior to enrolment in the study. Adobe sign feature on iPad or smartphone will be used in order to minimise the safety concerns for study personnel conducting the consent procedures. There is also be a separate section in the consent that allows the subjects to opt in or out of additional blood collection for ancillary studies in the future that are directly related to the current study.

The forms and the consenting process have been reviewed and approved by the Tulane IRB.

\section{Dissemination}

The data generated in this study will be presented at internal medicine, infections disease and endocrinology national or international conferences and published in a timely fashion. All final peer-reviewed manuscripts that arise from this proposal will be submitted to the digital archive PubMed Central. Results will be published in open-access journals when possible.

Study protocol and statistical analysis plans will be publically available. Participant-level data will not be shared.

\section{DISCUSSION}

The pathogenesis of COVID-19 involves an inappropriate immune response, with outpouring of proinflammatory chemokines, leading to lung infiltration and hyperactivation of monocytes and macrophages producing proinflammatory cytokines (cytokine storm), resulting in lung oedema, reduced gas exchange and ultimately leading 
to acute respiratory distress syndrome and multiorgan failure. ${ }^{9} 1028-33$ Repurposing approved drugs that have already been tested in humans-and for which detailed information is available on their pharmacology, formulation, dose and potential toxicity - provides a fast and safe approach for off-label use of potentially life-saving therapeutics. The scientific premise for the use of $\mathrm{E} 2$ and $\mathrm{P} 4$ in hospitalised patients, and its relevance to COVID-19 pathogenesis, is based on a large body of published literature reviewed recently. ${ }^{25}$ In fact, two clinical trials are testing E2 (ClinicalTrials.gov identifier NCT04359329) or P4 (ClinicalTrials.gov identifier NCT04365127) individually in COVID-19 patients. While this protocol was in review, the trial with $\mathrm{P} 4$ reported that in men hospitalised with moderate to severe COVID-19, a 5-day progesterone treatment resulted in three fewer days of supplemental oxygen need and 2.5 fewer days of hospital stay as compared with control subjects. ${ }^{34}$ The present study has been reviewed by the US Food and Drug Administration for Investigational New Drug authorisation since August 2020, which has delayed its beginning.

The advantage of repurposing E2 and P4 is the depth of knowledge regarding their clinical efficacy and toxicity that has accumulated from over half a century of clinical and basic research. Hormone therapy using oestrogens and progestogens is used by millions of women for contraception or the prevention of menopausal symptoms. There is no acute toxicity. The risk of DVT or pulmonary embolism associated with oestradiol and progesterone treatment exists but is minimal. First, the risk of DVT is associated with chronic hormone therapy, over months or years, not following acute hormone therapy of several days. Most importantly, the risk is minimal because of parenteral oestradiol administration, which does not alter clotting factors. DVT risk occurs mainly following orally administered oestrogens and synthetic progestins, because of first-pass liver metabolism producing high liver exposures to hormones, increasing hepatic production of clotting and inflammatory factors. ${ }^{35}$ Additionally, the DVT risks are usually associated with the use of conjugated equine oestrogens alone or associated with a synthetic progestin, like medroxyprogesterone acetate. ${ }^{35}$ There is no documented risk associated with natural E2 given systemically (intramuscularly, subcutaneously) and no documented risk with the use of natural P4. Finally, the pathogenesis of thrombosis in COVID-19 is a consequence of the immunoinflammation, ${ }^{36}$ and is expected to be ameliorated by the $\mathrm{E} 2$ and $\mathrm{P} 4$ treatment.

\section{Trial status}

This trial is currently recruiting subjects.

\section{Author affiliations}

${ }^{1}$ Section of Endocrinology and Metabolism, John W. Deming Department of Medicine, Tulane University Health Sciences Center, New Orleans, Louisiana, USA ${ }^{2}$ Section of Endocrinology, Department of Medicine, Southeast Louisiana Veterans Health Care System, New Orleans, Louisiana, USA

${ }^{3}$ Tulane Center of Excellence in Sex-Based Biology \& Medicine, Tulane University Health Sciences Center, New Orleans, Louisiana, USA
${ }^{4}$ Section of General Internal Medicine and Geriatrics, John W. Deming Department of Medicine, Tulane University School of Medicine, New Orleans, Louisiana, USA ${ }^{5}$ Clinical Translational Unit, Tulane University School of Medicine, New Orleans, Louisiana, USA

${ }^{6}$ Department of Biostatistics and Data Science, Tulane University School of Public Health and Tropical Medicine, New Orleans, Louisiana, USA

Acknowledgements We acknowledge the members of the DSMB: Frank Greenway, MD, DSMB Chair (Endocrinology) The Pennington Biomedical Research Center, Erin Brewer, MD, MPH (Women's Health) Southeast Louisiana Veterans Health Care System (SLVHCS), Phillip Yeon, MD, MPHTM (Infectious Diseases) SLVHCS, Nancy Vander Velde, MD (Hematology and Oncology) SLVHCS and Donald E. Mercante, PhD (Biostatistics) Louisiana State University.

Contributors FM-J: study design, management, analysis, interpretation of data; writing of the report; and decision to submit the report for publication. DL: clinical trial preparatory activities, data management, statistical analysis, interpretation of data and writing of the report. KB: recruitment of patients, interpretation of data and writing of the report. VAF: interpretation of data and writing of the report. JL: statistical analysis, interpretation and writing of the report. MS: visualisation and writing of the report.

Funding The trial is funded by the Center of Excellence in Sex-Based Biology \& Medicine, Tulane University, New Orleans, Louisiana, USA. FM-J is supported by National Institutes of Health awards (DK074970 and DK107444), an American Diabetes Association COVID-19 Research Award (7-20-COVID-051), and a US Department of Veterans Affairs Merit Review Award (BX003725). JL is supported in part by U54 GM104940 from the National Institute of General Medical Sciences of the National Institutes of Health, which funds the Louisiana Clinical and Translational Science Center.

Disclaimer The content is solely the responsibility of the authors and does not necessarily represent the official views of the National Institutes of Health.

Competing interests None declared.

Patient and public involvement Patients and/or the public were not involved in the design, or conduct, or reporting, or dissemination plans of this research.

Patient consent for publication Not applicable.

Provenance and peer review Not commissioned; externally peer reviewed.

Open access This is an open access article distributed in accordance with the Creative Commons Attribution Non Commercial (CC BY-NC 4.0) license, which permits others to distribute, remix, adapt, build upon this work non-commercially, and license their derivative works on different terms, provided the original work is properly cited, appropriate credit is given, any changes made indicated, and the use is non-commercial. See: http://creativecommons.org/licenses/by-nc/4.0/.

ORCID iD

Dragana Lovre http://orcid.org/0000-0002-2607-8768

\section{REFERENCES}

1 John Hopkins coronavirus resource center. cases by country. Available: https://gisanddata.maps.arcgis.com/apps/opsdashboard/ index.html\#/bda7594740fd40299423467b48e9ecf6 [Accessed 12 Nov 2021].

2 Khubchandani J, Sharma S, Price JH, et al. COVID-19 vaccination Hesitancy in the United States: a rapid national assessment. $J$ Community Health 2021;46:270-7.

3 RECOVERY Collaborative Group, Horby P, Lim WS, et al. Dexamethasone in hospitalized patients with Covid-19. N Engl J Med 2021;384:693-704.

4 Kaka AS, MacDonald R, Greer N. Major update: remdesivir for adults with COVID-19: a living systematic review and meta-analysis for the American College of physicians practice points. Ann Intern Med 2021;174:663-72.

5 Salvarani C, Dolci G, Massari M, et al. Effect of tocilizumab vs standard care on clinical worsening in patients hospitalized with COVID-19 pneumonia: a randomized clinical trial. JAMA Intern Med 2021;181:24-31.

6 Janiaud P, Axfors C, Schmitt AM, et al. Association of convalescent plasma treatment with clinical outcomes in patients with COVID-19: a systematic review and meta-analysis. JAMA 2021;325:1185-95.

7 Mehta SR, Yusuf S, Díaz R, et al. Effect of glucose-insulin-potassium infusion on mortality in patients with acute ST-segment elevation 
myocardial infarction: the CREATE-ECLA randomized controlled trial. JAMA 2005;293:437-46.

8 Chen G, Wu D, Guo W, et al. Clinical and immunological features of severe and moderate coronavirus disease 2019. J Clin Invest 2020;130:2620-9.

9 Huang C, Wang Y, Li X, et al. Clinical features of patients infected with 2019 novel coronavirus in Wuhan, China. Lancet 2020;395:497-506.

10 Blanco-Melo D, Nilsson-Payant BE, Liu W-C, et al. Imbalanced host response to SARS-CoV-2 drives development of COVID-19. Cell 2020;181:1036-45.

11 Chousterman BG, Swirski FK, Weber GF. Cytokine storm and sepsis disease pathogenesis. Semin Immunopathol 2017;39:517-28.

12 Ye Q, Wang B, Mao J. The pathogenesis and treatment of the Cytokine Storm in COVID-19. J Infect 2020;80:607-13.

13 Tanaka T, Narazaki M, Kishimoto T. Immunotherapeutic implications of IL-6 blockade for cytokine storm. Immunotherapy 2016;8:959-70.

14 McGonagle D, Sharif K, O'Regan A, et al. The role of cytokines including interleukin-6 in COVID-19 induced pneumonia and macrophage activation syndrome-like disease. Autoimmun Rev 2020;19:102537.

15 Guan W-J, Ni Z-Y, Hu Y, et al. Clinical characteristics of coronavirus disease 2019 in China. N Engl J Med 2020;382:1708-20.

16 Klein SL, Dhakal S, Ursin RL, et al. Biological sex impacts COVID-19 outcomes. PLoS Pathog 2020;16:e1008570.

17 Mauvais-Jarvis F. Aging, male sex, obesity, and metabolic inflammation create the perfect storm for COVID-19. Diabetes 2020;69:1857-63.

18 Mauvais-Jarvis F, Bairey Merz N, Barnes PJ, et al. Sex and gender: modifiers of health, disease, and medicine. Lancet 2020;396:565-82.

19 Richardson S, Hirsch JS, Narasimhan M, et al. Presenting characteristics, comorbidities, and outcomes among 5700 patients hospitalized with COVID-19 in the New York City area. JAMA 2020;323:2052-9.

20 Scully EP, Haverfield J, Ursin RL, et al. Considering how biological sex impacts immune responses and COVID-19 outcomes. Nat Rev Immunol 2020;20:442-7.

21 Klein SL, Flanagan KL. Sex differences in immune responses. Nat Rev Immunol 2016;16:626-38.
22 Seeland U, Coluzzi F, Simmaco M, et al. Evidence for treatment with estradiol for women with SARS-CoV-2 infection. BMC Med 2020;18:369.

23 Phiel KL, Henderson RA, Adelman SJ, et al. Differential estrogen receptor gene expression in human peripheral blood mononuclear cell populations. Immunol Lett 2005;97:107-13.

24 Straub RH. The complex role of estrogens in inflammation. Endocr Rev 2007;28:521-74.

25 Mauvais-Jarvis F, Klein SL, Levin ER. Estradiol, progesterone, immunomodulation, and COVID-19 outcomes. Endocrinology 2020;161 doi:10.1210/endocr/bqaa127

26 World Health Organization R\&D blueprint novel coronavirus COVID-19 therapeutic trial synopsis. Available: https://www.who.int/ teams/blueprint/covid-19 [Accessed 25 Apr 2021].

27 O'Brien PC, Fleming TR. A multiple testing procedure for clinical trials. Biometrics 1979;35:549-56.

28 Mehta P, McAuley DF, Brown M, et al. COVID-19: consider cytokine storm syndromes and immunosuppression. Lancet 2020;395:1033-4.

29 Chen G, Wu D, Guo W, et al. Clinical and immunological features of severe and moderate coronavirus disease 2019. J Clin Invest 2020;130:2620-9.

30 Chousterman BG, Swirski FK, Weber GF. Cytokine storm and sepsis disease pathogenesis. Semin Immunopathol 2017;39:517-28.

31 Ye Q, Wang B, Mao J. The pathogenesis and treatment of the cytokine storm in COVID-19. J Infect 2020.

32 Tanaka T, Narazaki M, Kishimoto T. Immunotherapeutic implications of IL-6 blockade for cytokine storm. Immunotherapy 2016;8:959-70.

33 McGonagle D, Sharif K, O'Regan A, et al. The role of cytokines including interleukin-6 in COVID-19 induced pneumonia and macrophage activation syndrome-like disease. Autoimmun Rev 2020;19:102537.

34 Ghandehari S, Matusov Y, Pepkowitz S, et al. Progesterone in addition to standard of care vs standard of care alone in the treatment of men hospitalized with moderate to severe COVID-19: a randomized, controlled pilot trial. Chest 2021;160:74-84.

35 Mauvais-Jarvis F, Manson JE, Stevenson JC, et al. Menopausal hormone therapy and type 2 diabetes prevention: evidence, mechanisms, and clinical implications. Endocr Rev 2017;38:173-88.

36 Bonaventura A, Vecchié A, Dagna L. Endothelial dysfunction and immunothrombosis as key pathogenic mechanisms in COVID-19. Nat Rev Immunol 2021:1-11. 